\title{
Nuclear pore complex during neuronal degeneration
}

\section{Cracking the last barrier!}

\author{
Daniele Bano, ${ }^{1,2, *}$ Michael O. Hengartner ${ }^{3}$ and Pierluigi Nicotera ${ }^{1, *}$ \\ ${ }^{1}$ Deutsches Zentrum für Neurodegenerative Erkrankungen (DZNE); Bonn, Germany; ${ }^{2} \mathrm{MRC}$ Toxicology Unit; University of Leicester; Leicester, UK; \\ ${ }^{3}$ Institute of Molecular Biology; University of Zurich; Zurich, Switzerland
}

Key words: calcium, calpains, C. elegans, excitotoxicity, nucleoporins, nucleus

Submitted: $10 / 15 / 09$

Revised: 11/22/09

Accepted: 11/29/09

Previously published online: www.landesbioscience.com/journals/ nucleus/article/10798

${ }^{*}$ Correspondence to:

Daniele Bano and Pierluigi Nicotera; Email: daniele. bano@dzne.de and pierluigi.nicotera@dzne.de
Tn eukaryotic cells, the exchange of molecules between the genetic material within the nucleus and the cytosol occurs through the Nuclear Pore Complex (NPC), which is a large membrane-embedded assembly composed by multiple proteins named nucleoporins arranged around an aqueous channel. The bi-directional passive diffusion and the active transport of factors across the nuclear envelope are responsible for a variety of biological processes and they are controlled respectively by the size of the pore and the interaction between nucleoporins and karyopherins. Thus, it is not surprising that most of the degenerative programs induce cellular stress by altering the NPC composition or the binding between nucleoporins and docking factors. This facilitates the access of nuclear DNA to pro-death factors, amplify the detrimental cascade and finally play a role in the disassembly of the nuclear structure. Recently, we have shown that during calciummediated neuronal degeneration NPC components can be degraded with consequent increase of NPC channel permeability. Moreover, we proved that these changes occurred much earlier than the final disassembly of the nuclear envelope and they are mediated by calcium overload. Is the increase of NPC leakiness the executioner of the excitotoxic process or simply a final event of a cell condemned to death? Here we speculate the consequence of the nucleoporin loss, the alteration of nucleocytoplasmic transport and their contribution to neuronal demise.

\section{Introduction}

In eukaryotic cells, the exchange of information between the genetic material within the nucleus and the cytoplasm is required for the coordination of cellular processes. ${ }^{1}$ The movement of macromolecules across the nuclear envelope occurs through the aqueous channel of the NPC, which is composed by multiple copies of about 30 different proteins named nucleoporins responsible for the rigid core scaffold and the selective barrier of the pore. ${ }^{2}$ Essentially, the NPC is composed by five distinct assemblies of nucleoporins on the basis to their locations: the membrane, the outer, the inner and the linker rings and the phenylalanine-glycine (FG) nucleoporins. The first three substructures form the NPC core and anchor the structure to the nuclear envelope, whereas unfolded and clustered regions of FG nucleoporins fill the central channel of the NPC, restrict the passage of molecules through the pore and allow highly selective association with nuclear transport receptors and their cargos. ${ }^{3,4}$ The free diffusion is restricted to molecules smaller than $40-50 \mathrm{kDa}$, whereas the transit of large cargo-complexes through the NPC resealing structure is energy dependent and actively regulated. ${ }^{5}$ Moreover, dynamic changes of NPC global structure, including the complete disassembly of the complex, and alteration of nucleoporin composition can occur as essential requirements for the coordination of cellular functions during the different stage of the cell cycle. ${ }^{6,7}$ Clearly, any alteration affecting nuclear transport or the organization 


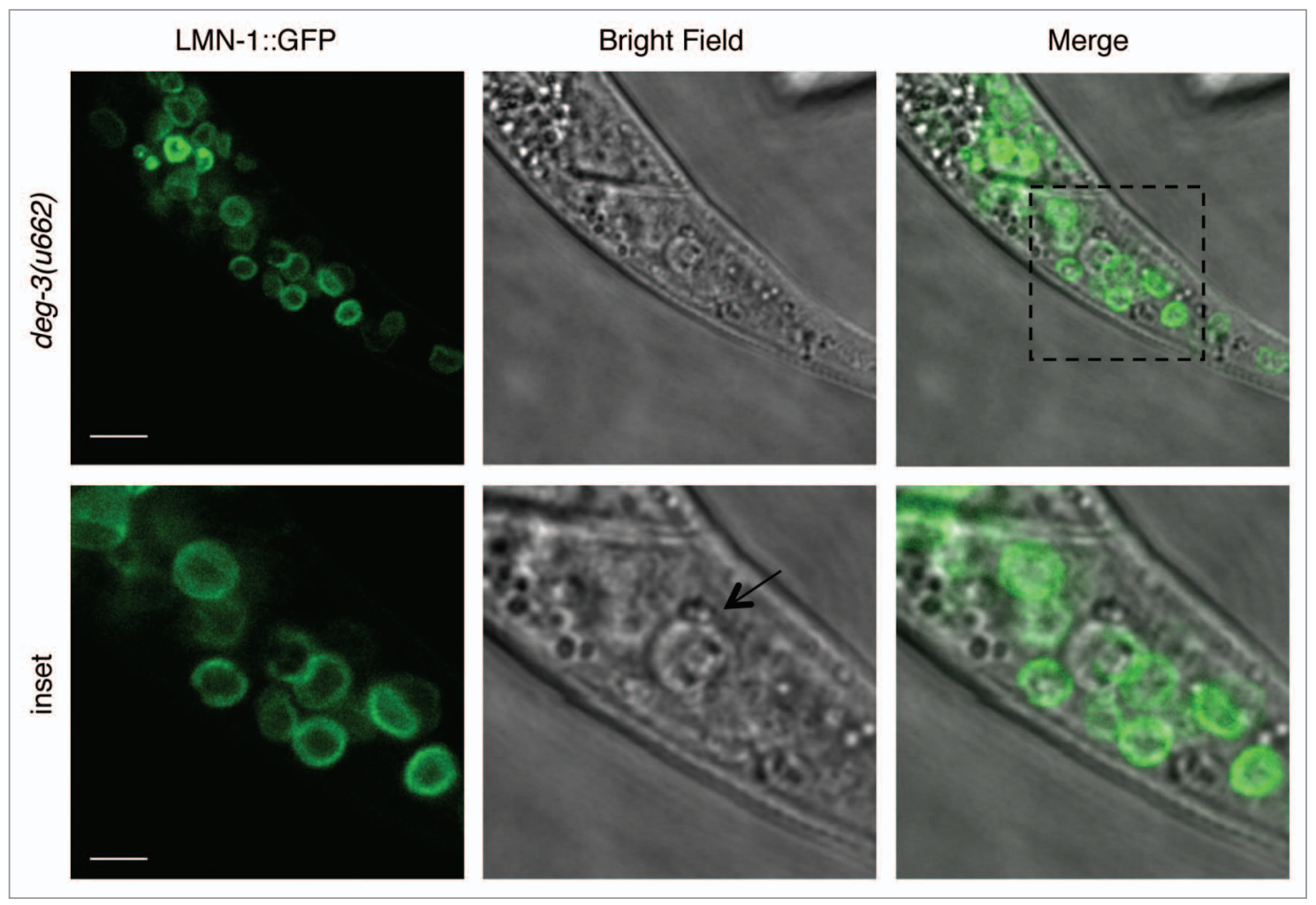

Figure 1. Confocal analysis of deg-3(u662) worms carrying the $P_{l m n-1}:: I m n-1:: G F P$ transgene. Degenerating neuron (arrow) presented partial loss of GFP signal at the nuclear envelope (size bar upper $=10 \mu \mathrm{m}$; size bar inset $=5 \mu \mathrm{m}$ ).

of NPC would alter the permeability of the nuclear envelope, with a significant impact on DNA transcriptional regulation and therefore on a range of cellular processes, such as in the case of viral infection. Indeed, several studies have shown that a variety of trafficking pathways are perturbed in virus-infected cells, with an increase leakiness of the nuclear envelope caused by the degradation of some nucleoporins, which facilitates the shuttling of viral macromolecules and therefore viral replication. ${ }^{8}$ Similarly, during apoptosis structural changes of the nuclear envelope and NPC ultimately trigger the re-distribution of pro-apoptotic factors and the consequent amplification of the death-signal., ${ }^{90}$ Several proteins have been described to translocate from the cytoplasm to the nucleus during apoptosis, including caspases, ${ }^{11,12}$ which cleave nuclear proteins such as lamins and nucleoporins, Apaf- $1{ }^{13-15}$ the death receptor adaptor protein TRADD ${ }^{16}$ and the mitochondrial proteins Cytochrome $C$ and Apoptosis Inducing Factor (AIF). ${ }^{17}$ In this context, reduction of nuclear trafficking by wheat germ agglutinin (WGA) or antibodies to importins can prevent Fas-induced apoptosis, ${ }^{18}$ as well as viral replication, ${ }^{19}$ which suggests that access to the nucleus is a prerequisite for the coordination of the death program. Thus, crosstalk between the nucleus and the other intracellular compartments is tightly coordinated and implicated in a variety of pathological processes. What is the role of the NPC during neuronal demise? Is the NPC a regulatory player or a simple viewer of the show?

In the last decade, a growing number of groups have reported that several proteins accumulate in the nucleus of neurons under a variety of stressing conditions and this contributes to cytotoxicity. Importantly, pharmacological or genetic treatments that suppress the improper translocation of factors into the nucleus, such as in the case of $\mathrm{GAPDH}^{20,21}$ or HDAC $4{ }^{22}$ can promote neuronal survival, which means that improper localization of proteins can influence cell death cascade and therefore determine cell fate. We have recently studied the changes in protein localization during excitotoxicity, a process triggered by excessive glutamate release that induces extensive membrane depolarization and neuronal degeneration. We have shown that elevation of calcium facilitates the re-distribution of smallsize proteins across the nuclear envelope. Furthermore, calcium-activated proteases, calpains, increase the permeability of the NPC by altering NPC components with consequent impairment of the nuclear transport machinery. ${ }^{23}$ Although calpains are involved in the disassembly of the nucleus (Fig. 1), the detachment of the heterochromatin from the nuclear envelope and the disassembly of the NPC occur much later than the re-distribution of molecules. Thus, our findings shed light on an unknown hierarchical sequence of events, which takes place during caspaseindependent cell death and is required for the onset of nuclear disruption. Changes in free cytosolic $\mathrm{Ca}^{2+}$ can affect the nuclear accumulation of calmodulin, ${ }^{24}$ which binds to and modulates the activity of a large number of enzymes, and the subcellular localization of other cytosolic proteins, such as the cytoplasmic components of NFAT transcriptional complexes, ${ }^{25}$ the transcriptional factors Crz1, ${ }^{26}$ and various MAP and CaM kinases, phosphatases 
and phospholipases. ${ }^{27}$ In this scenario, it is plausible that sustained calcium rise as during excitotoxicity could trigger the concomitant activation of a wide range of transcriptional machinery and therefore a series of overlapping pathways. Thus, consistent with previous findings, our data suggest that sustained calcium deregulation affects the localization of proteins across the nuclear envelope and might represent a switch from the tight and regulated processing of genetic information to a less restricted access to nuclear DNA.

Currently, it is largely unclear whether blocking the NPC permeability by overexpression of non-cleavable nucleoporin mutants or injection of WGA can prevent neuronal loss during excitotoxicity. Nevertheless, as regulation of nuclear shuttling is neuroprotective in several cell death subroutines and since calpain inhibitors can delay calcium-mediated cell demise, ${ }^{28,29}$ it is tempting to speculate that increased permeability of the NPC is the "point of not return" that directly influences neurotoxicity induced by alteration of calcium homeostasis. What are the consequences of nucleoporin loss? Alteration in the expression and in the activity of NPC components can cause disease such as myelodysplastic syndrome, ${ }^{30}$ lead to increase DNA damage ${ }^{31}$ and impair homologous recombination and SUMOdependent DNA repairing. ${ }^{32}$ Interestingly, in differentiated cells, oxidation of a subset of long-lived nucleoporins dramatically decreases NPC turnover, which enhances nuclear leakiness and could be responsible for age-related events. ${ }^{33,34}$ The structural damage of the functional barrier caused by the degradation of nucleoporins and the nuclear accumulation of the proteases normally restricted to the cytosol would contribute to the passage of pro-death factors across the nuclear envelope and promote the onset of the degenerating cascade. Whether spatio-temporal activation of calpains can have a physiological role in the regulation of NPC activity in healthy cells during the cell cycle remains a further challenging question for future investigations.

In summary, because in most neurodegenerative disorders neurons lose the ability to tightly control $\mathrm{Ca}^{2+}$ homeostasis, ${ }^{35,36}$ it is tempting to speculate that sustained calcium deregulation can promote detrimental processes and elicit neuronal degeneration by altering a wide range of targets, including NPC permeability and nucleocytoplasmic trafficking. Pharmacological inhibitors that prevent redistribution of molecules across the nuclear envelope might prevent deregulation of transcriptional activity and therefore can be beneficial against neurotoxicity.

\section{References}

1. Tran EJ, Wente SR. Dynamic nuclear pore complexes: life on the edge. Cell 2006; 125:1041-53.

2. Alber F, Dokudovskaya S, Veenhoff LM, Zhang W, Kipper J, Devos D, et al. The molecular architecture of the nuclear pore complex. Nature 2007; 450:695701

3. Frey S, Richter RP, Gorlich D. FG-rich repeats of nuclear pore proteins form a three-dimensional meshwork with hydrogel-like properties. Science (New York, NY) 2006; 314:815-7.

4. Lim RY, Fahrenkrog B, Koser J, Schwarz-Herion K, Deng J, Aebi U. Nanomechanical basis of selective gating by the nuclear pore complex. Science (New York, NY) 2007; 318:640-3.

5. Cook A, Bono F, Jinek M, Conti E. Structural biology of nucleocytoplasmic transport. Annu Rev Biochem 2007; 76:647-71.

6. Maul GG, Maul HM, Scogna JE, Lieberman MW, Stein GS, Hsu BY, et al. Time sequence of nuclear pore formation in phytohemagglutinin-stimulated lymphocytes and in HeLa cells during the cell cycle. J Cell Biol 1972; 55:433-47.

7. Harel A, Forbes DJ. Importin beta: conducting a much larger cellular symphony. Mol Cell 2004; $16: 319-30$

8. Gustin KE, Sarnow P. Effects of poliovirus infection on nucleo-cytoplasmic trafficking and nuclear pore complex composition. EMBO J 2001; 20:240-9.

9. Ferrando-May E, Cordes V, Biller-Ckovric I, Mirkovic J, Gorlich D, Nicotera P. Caspases mediate nucleoporin cleavage, but not early redistribution of nuclear transport factors and modulation of nuclear permeability in apoptosis. Cell Death Differ 2001; 8:495-505

10. Ferrando-May E. Nucleocytoplasmic transport in apoptosis. Cell Death Differ 2005; 12:1263-76.

11. Paroni G, Henderson C, Schneider C, Brancolini C. Caspase-2 can trigger cytochrome $C$ release and apoptosis from the nucleus. J Biol Chem 2002; 277:15147-61.

12. Faleiro L, Lazebnik Y. Caspases disrupt the nuclearcytoplasmic barrier. J Cell Biol 2000; 151:951-9.

13. Chen F, Hersh BM, Conradt B, Zhou Z, Riemer D, Gruenbaum Y, et al. Translocation of C. elegans CED-4 to nuclear membranes during programmed cell death. Science 2000; 287:1485-9.

14. Sakai T, Liu L, Teng X, Mukai-Sakai R, Shimada H, Kaji R, et al. Nucling recruits Apaf-1/pro-caspase-9 complex for the induction of stress-induced apoptosis. J Biol Chem 2004; 279:41131-40.

15. Zermati Y, Mouhamad S, Stergiou L, Besse B, Galluzzi L, Boehrer S, et al. Nonapoptotic role for Apaf-1 in the DNA damage checkpoint. Mol Cell 2007; 28:624-37.

16. Morgan M, Thorburn J, Pandolfi PP, Thorburn A. Nuclear and cytoplasmic shuttling of TRADD induces apoptosis via different mechanisms. J Cell Biol 2002; 157:975-84.

17. Susin SA, Lorenzo HK, Zamzami N, Marzo I, Snow $\mathrm{BE}$, Brothers GM, et al. Molecular characterization of mitochondrial apoptosis-inducing factor. Nature 1999; 397:441-6.
18. Yasuhara N, Eguchi Y, Tachibana T, Imamoto N, Yoneda Y, Tsujimoto Y. Essential role of active nuclear transport in apoptosis. Genes Cells 1997; 2:55-64.

19. Rabe B, Vlachou A, Pante N, Helenius A, Kann M. Nuclear import of hepatitis B virus capsids and release of the viral genome. Proc Natl Acad Sci USA 2003; 100:9849-54.

20. Hara MR, Agrawal N, Kim SF, Cascio MB, Fujimuro M, Ozeki Y, et al. S-nitrosylated GAPDH initiates apoptotic cell death by nuclear translocation following Siah1 binding. Nat Cell Biol 2005; 7:665-74.

21. Hara MR, Thomas B, Cascio MB, Bae BI, Hester LD, Dawson VL, et al. Neuroprotection by pharmacologic blockade of the GAPDH death cascade. Proc Natl Acad Sci USA 2006; 103:3887-9.

22. Bolger TA, Yao TP. Intracellular trafficking of histone deacetylase 4 regulates neuronal cell death. J Neurosci 2005; 25:9544-53.

23. Bano D, Dinsdale D, Cabrera-Socorro A, Maida $\mathrm{S}$, Lambacher N, McColl B, et al. Alteration of the nuclear pore complex in $\mathrm{Ca}(2+)$-mediated cell death. Cell Death Differ 2009.

24. Liao B, Paschal BM, Luby-Phelps K. Mechanism of $\mathrm{Ca}^{2+}$-dependent nuclear accumulation of calmodulin. Proc Natl Acad Sci USA 1999; 96:6217-22.

25. Chow CW, Rincon M, Cavanagh J, Dickens M, Davis RJ. Nuclear accumulation of NFAT4 opposed by the JNK signal transduction pathway. Science (New York, NY) 1997; 278:1638-41.

26. Matheos DP, Kingsbury TJ, Ahsan US, Cunningham KW. Tcnlp/Crzlp, a calcineurin-dependent transcription factor that differentially regulates gene expression in Saccharomyces cerevisiae. Genes Dev 1997; 11:3445-58.

27. Carafoli E, Santella L, Branca DMB. Generation, control and processing of cellular calcium signals. Crit Rev Biochem Mol Biol 2001; 36:107-260.

28. Bano D, Young KW, Guerin CJ, Lefeuvre R, Rothwell NJ, Naldini L, et al. Cleavage of the plasma membrane $\mathrm{Na}^{+} / \mathrm{Ca}^{2+}$ exchanger in excitotoxicity. Cell 2005; 120:275-85.

29. Syntichaki P, Xu K, Driscoll M, Tavernarakis N. Specific aspartyl and calpain proteases are required for neurodegeneration in C. elegans. Nature 2002; 419:939-44.

30. Nishiyama M, Arai $Y$, Tsunematsu Y, Kobayashi $\mathrm{H}$, Asami K, Yabe M, et al. 11p15 translocations involving the NUP98 gene in childhood therapy-related acute myeloid leukemia/myelodysplastic syndrome. Genes Chromosomes Cancer 1999; 26:215-20.

31. Bennett CB, Lewis LK, Karthikeyan G, Lobachev $\mathrm{KS}$, Jin YH, Sterling JF, et al. Genes required for ionizing radiation resistance in yeast. Nat Genet 2001; 29:426-34.

32. Nagai S, Dubrana K, Tsai-Pflugfelder M, Davidson MB, Roberts TM, Brown GW, et al. Functional targeting of DNA damage to a nuclear pore-associated SUMO-dependent ubiquitin ligase. Science (New York, NY) 2008; 322:597-602.

33. Haithcock E, Dayani Y, Neufeld E, Zahand AJ, Feinstein N, Mattout A, et al. Age-related changes of nuclear architecture in Caenorhabditis elegans. Proc Natl Acad Sci USA 2005; 102:16690-5.

34. D’Angelo MA, Raices M, Panowski SH, Hetzer MW. Age-dependent deterioration of nuclear pore complexes causes a loss of nuclear integrity in postmitotic cells. Cell 2009; 136:284-95.

35. Berliocchi L, Bano D, Nicotera P. $\mathrm{Ca}^{2+}$ signals and death programmes in neurons. Philos Trans R Soc Lond B Biol Sci 2005; 360:2255-8.

36. Mattson MP, Chan SL, Duan W. Modification of brain aging and neurodegenerative disorders by genes, diet and behavior. Physiol Rev 2002; 82:637-72. 